\title{
Efficacy of immunomodulation in the treatment of profound thrombocytopenia after adult cardiac surgery
}

\author{
J. Scott Rankin, MD, and Charles W. Stratton, MD
}

\begin{abstract}
Objective: Causes of profound thrombocytopenia (platelet count $<60 \mathrm{~K}$ ) developing days after cardiac surgery include heparin platelet factor 4 antibodies, thrombotic thrombocytopenic purpura-like antibodies, and endotoxin generated by pulmonary infections. Modulation of immune-mediated profound thrombocytopenia with intravenous immunoglobulin could be efficacious for any of these conditions.
\end{abstract}

\begin{abstract}
Methods: From 2002 to 2010, profound thrombocytopenia developed in 20 consecutive patients within days after cardiac surgery; 19 patients underwent valve or aortic operations, and 1 patient underwent coronary bypass. Risk profiles were high preoperatively: Patients' mean age was 73 years, 50\% underwent nonelective procedures, $100 \%$ had comorbidities, and $25 \%$ underwent reoperations. When decreasing platelet counts approached $60 \mathrm{~K}$, intravenous immunoglobulin was started at $1.5 \mathrm{~g} / \mathrm{kg}$ intravenously over 5 days. Anticoagulation and platelet transfusions were avoided. In 1 patient, profound thrombocytopenia failed to reverse promptly, and daily plasmapheresis was introduced. Platelet counts before and after interventions were assessed with linear regression analyses over time, including a spline function and statistical knot coincident with starting intravenous immunoglobulin.
\end{abstract}

Results: In 19 of 20 patients, profound thrombocytopenia stabilized and rebounded within 2 to 4 days after initiating intravenous immunoglobulin. In the remaining slow-responding patient, addition of plasmapheresis was associated with rapid recovery. In every patient, coincident multiorgan failure reversed, and 19 of 20 patients recovered uneventfully and survived hospitalization with no limb ischemia or tissue loss. No complications of intravenous immunoglobulin therapy or plasmapheresis were observed.

Conclusions: Although mechanisms of profound thrombocytopenia after cardiac surgery are poorly understood, they likely relate to inappropriate autoimmune moieties causing peripheral platelet aggregation and multiorgan failure. A protocol involving immunomodulation with intravenous immunoglobulin supplemented by plasmapheresis appeared safe and efficacious. Direct immunologic interventions for profound thrombocytopenia could improve postoperative outcomes. (J Thorac Cardiovasc Surg 2014;147:808-15)

Profound thrombocytopenia (pT) occurring early postoperatively is observed in approximately $1 \%$ to $3 \%$ of patients after adult cardiac surgery. ${ }^{1}$ The disorder can be associated with diffuse intravascular platelet deposition, strokes, multiorgan failure, venous thrombi, pulmonary embolism, limb loss, and death. ${ }^{2}$ Although this syndrome is commonly called heparin-induced thrombocytopenia (HIT) in current practice, it is likely that true heparin-associated platelet factor $4(\mathrm{PF} 4)$ antibodies are etiologic in only a minority of patients. ${ }^{1}$ Other likely causes for postoperative platelet aggregation include thrombotic thrombocytopenic purpura

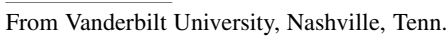

Disclosures: Dr Rankin reports consulting fees from and equity ownership in BioStable Science and Engineering. All other authors have nothing to disclose with regard to commercial support.

Read at the 93rd Annual Meeting of The American Association for Thoracic Surgery, Minneapolis, Minnesota, May 4-8, 2013.

Received for publication April 29, 2013; revisions received Sept 1, 2013; accepted for publication Sept 25, 2013; available ahead of print Nov 18, 2013.

Address for reprints: J. Scott Rankin, MD, Department of Cardiac Surgery, Vanderbilt University, 320 Lynnwood Blvd, Nashville, TN 37205 (E-mail: jsrankinmd@cs. com).

$0022-5223 / \$ 36.00$

Copyright (c) 2014 by The American Association for Thoracic Surgery

http://dx.doi.org/10.1016/j.jtcvs.2013.09.051
(TTP)-like antibodies, ${ }^{3}$ infectious superantigens, ${ }^{4,5}$ and gram-negative endotoxin. ${ }^{6,7}$ Whatever the cause, most agree that surgical $\mathrm{pT}$ is mediated by immune activation/ dysfunction producing inappropriate autoantibodies and peripheral platelet aggregation. The current therapeutic standard probably is anticoagulation with direct thrombin inhibitors, ${ }^{8}$ although plasmapheresis for removal of autoantibodies recently has shown promise. ${ }^{9}$

Many types of immune-mediated thrombocytopenia exist in general medical practice. ${ }^{6}$ In recent years, another direct immune intervention, immune modulation using intravenous immunoglobulin (IVIG), has become a primary therapy for many of these disorders. ${ }^{10}$ IVIG contains active normal human antibodies from more than 10,000 donors and is highly effective in modulating immune dysfunction and "normalizing" pathologic immunologic moieties. In fact, treatment of "acute immune-mediated thrombocytopenia" currently is an on-label indication for IVIG. ${ }^{11}$ Because postoperative $\mathrm{pT}$ seems to be primarily immune mediated, IVIG also could be useful in the postsurgical setting. This article reports the results of treating $\mathrm{pT}$ with immune modulation using IVIG. Many of these concepts have been published, ${ }^{12}$ and the purpose of this 

Abbreviations and Acronyms
HIT = heparin-induced thrombocytopenia
Ig = immunoglobulin
IVIG $=$ intravenous immunoglobulin
PF4 = platelet factor 4
pT $=$ profound thrombocytopenia
TTP $=$ thrombotic thrombocytopenic purpura

communication is to present specific pT data and associated discussion.

\section{MATERIALS AND METHODS \\ Ethical Considerations}

This clinical effort to use IVIG for the purpose of treating $\mathrm{pT}$ after adult cardiac surgery was initiated within a standard cardiac surgical practice in the setting of a worsening high-risk problem with less than satisfactory solutions. One could reason that immune modulation for pT was a Food and Drug Administration on-label indication for IVIG ${ }^{11}$ and the concept was based on existing literature support in similar clinical situations. ${ }^{13-16}$ Before IVIG was started, possible therapeutic benefits and risks were discussed with the patients and families, and all agreed to proceed. The chart review and de-identified data analysis were performed at a later date under a waiver of informed consent by the Western Institutional Review Board.

\section{Population}

Twenty consecutive patients experiencing $\mathrm{pT}$ after adult cardiac surgery from 2002 to the middle of 2010 were assessed. The first 14 patients were part of a previous report on general immune dysfunction from 2002 to the end of $2009,{ }^{12}$ and the final 6 patients were from the subsequent half-year. Nineteen patients underwent valve or aortic operations, and 1 patient underwent coronary bypass. Detailed characteristics of the overall immune dysfunction population have been described, ${ }^{12}$ and for the patients experiencing pT in this analysis, specific data are provided in Table 1. Risk profiles were high: Patients' average age was $73.0 \pm 15.6$ years (mean \pm standard deviation), $50 \%$ underwent nonelective procedures, $100 \%$ had comorbidities, $25 \%$ underwent reoperations, and more than half were female. Criteria for diagnosis of pT included a dramatically decreasing platelet count days after adult cardiac surgery, averaging a $20 \mathrm{~K}$ decrease the day before starting IVIG (Figure 1), with no other cause evident, and with the platelet count approaching $60 \mathrm{~K}$. Most patients had pulmonary infiltrates associated with pulmonary dysfunction, leukocytosis, and some degree of multiorgan failure at the time of pT development, and the few who did not eventually developed pulmonary infiltrates within days. Postoperative immunoglobulin (Ig)G levels were low in most (Table 1$),{ }^{12}$ but were only available later in the series. Heparin PF4 antibody tests were not available early in the series, but when obtained in later patients, all results were negative.

\section{General Management Protocol}

Most patients with pT had evidence of pulmonary infection with worsening pulmonary infiltrates, signs of sepsis, leukocytosis, deterioration of pulmonary mechanics, and impairment of pulmonary gas exchange. ${ }^{12}$ Multiple-drug intravenous antibiotics (consisting of combinations of piperacillin/tazobactam, cefepime, tobramycin, and meropenem [vancomycin was used only for specific indications]) were started in all patients, according to the University of Kentucky antibiotic protocol for management of hospital-acquired pneumonia. ${ }^{17,18}$ Platelet transfusions and anticoagulation with thrombin inhibitors were avoided. All patients were maintained under full intensive care unit care and had documentation of good cardiac status with a Swan-Ganz catheter and a transthoracic echocardiogram at the outset. Pulmonary cultures were obtained in all patients, including bronchial washings or protected brush cultures in patients undergoing therapeutic fiberoptic bronchoscopy. Significant renal dysfunction also developed in $45 \%$ of patients, manifested by oliguria or aneuria with increasing creatinine, despite intravenous dopamine and diuretic support. ${ }^{12}$

\section{Intravenous Immunoglobulin Therapy}

When the rapidly decreasing platelet count approached $60 \mathrm{~K}$ (Figure 1) and after family agreement, IVIG therapy (Carimune; ZLB Behring Inc, King of Prussia, Pa) was begun at a dose of 18 or $24 \mathrm{~g} / \mathrm{d}$ (depending on the size of the patient, $\sim 0.3 \mathrm{~g} / \mathrm{kg} / \mathrm{d}$ ) intravenously for 5 days to a total dose of $1.5 \mathrm{~g} / \mathrm{kg}$. In the subsequent spline function analysis, the first day of IVIG infusion was considered IVIG day 0 for placement of a statistical knot. More detailed clinical management principles have been outlined by Rankin and colleagues, ${ }^{12}$ including the importance of early enteral nutritional support. However, with IVIG therapy, most patients rapidly regained their appetites and promptly resumed good oral nutrition.

\section{Analysis Protocol}

In all patients, platelet counts were obtained almost daily as part of routine postoperative care. At a later time, retrospective chart review was performed, and platelet values were documented for 3 days before (day -3 ) beginning IVIG therapy (on day 0 ) and for 5 days afterward $($ day +5$)$. For a variety of reasons in the clinical setting, 18 of the total 180 platelet count data points ( 9 data points $\times 20$ patients) were missing, and these were interpolated by using the arithmetic average of adjacent data points. In the 20 patients, full IVIG therapy was initiated between the second and fifth postoperative days. When IVIG was started on postoperative day 2 , the preoperative platelet count was used as the day -3 data point. By using each patient as his or her own control, the therapeutic effect of IVIG was assessed with linear regression analysis of platelet counts over time using a spline function and a statistical knot at day 0 , coincident with beginning IVIG.

\section{RESULTS}

Most patients had signs of pulmonary infection coincident with pT. Three patients presented initially with isolated pT, but pulmonary infiltrates eventually developed. Thus, postoperative nosocomial infection was the probable cause in most patients, if not all. No patients had other complications or evidence of alternative sources of infection, and no other patients during this period had pT treated differently or any evidence of limb ischemia. At the time of IVIG initiation, most patients' conditions were deteriorating, with rapidly decreasing platelet counts (Figure 1), worsening ventilator status, and developing multiorgan failure. ${ }^{12}$ In the linear regression analysis over the 3 days before IVIG, the platelet counts were decreasing rapidly, and when the decision for IVIG was made (day 0 ), general morbidity often was high. Postoperative IgG levels were usually low in this population (Table 1), although quantitative $\operatorname{IgG}$ levels were available only in later years. After beginning IVIG on day 0, improvement in clinical course and reversal of thrombocytopenia were observed uniformly over the subsequent 5 days. In the spline function analysis, and using each patient as his or her own control, 
TABLE 1. Patient characteristics

\begin{tabular}{|c|c|c|c|c|c|c|c|c|c|c|c|c|}
\hline $\begin{array}{c}\text { Patient } \\
\text { no. }\end{array}$ & $\begin{array}{c}\text { Age, } \\
\mathbf{y}\end{array}$ & Sex & $\begin{array}{c}\text { No. of } \\
\text { comorbidities }\end{array}$ & Reoperation & $\begin{array}{c}\text { Cause of } \\
\text { disease }\end{array}$ & $\begin{array}{c}\text { NYHA } \\
\text { angina } \\
\text { class }\end{array}$ & $\begin{array}{c}\text { NYHA } \\
\text { class for } \\
\text { congestive } \\
\text { heart } \\
\text { failure }\end{array}$ & $\begin{array}{c}\text { Preoperative } \\
\text { albumin } \\
\text { level }\end{array}$ & $\begin{array}{l}\text { Nonelective } \\
\text { presentation }\end{array}$ & Procedure & $\begin{array}{c}\text { Pump } \\
\text { time (h) }\end{array}$ & $\begin{array}{c}\text { Postoperative } \\
\text { IgG level }\end{array}$ \\
\hline 1 & 62 & M & 4 & $\mathrm{~N}$ & $\begin{array}{l}\text { Ischemic mitral } \\
\text { disease }\end{array}$ & 4 & 1 & 3.4 & $\mathrm{Y}$ & AM-VR, $C \times 4$ & 4.08 & - \\
\hline 2 & 69 & M & 2 & $\mathrm{~N}$ & Mitral prolapse & 1 & 4 & 2 & $\mathrm{Y}$ & MT-VR & 3.35 & - \\
\hline 3 & 78 & $\mathrm{M}$ & 4 & $\mathrm{~N}$ & Mitral prolapse & 1 & 4 & 3.4 & $\mathrm{Y}$ & $\mathrm{M}-\mathrm{VR}, \mathrm{C} \times 2$ & 2.93 & - \\
\hline 4 & 69 & $\mathrm{~F}$ & 1 & $\mathrm{Y}$ & Mitral prolapse & 1 & 3 & 3.8 & $\mathrm{~N}$ & $\mathrm{M}-\mathrm{VR}, \mathrm{Abl}$ & 1.90 & - \\
\hline 5 & 81 & $\mathrm{~F}$ & 1 & $\mathrm{~N}$ & Calcific & 1 & 4 & 2.8 & $\mathrm{Y}$ & A-VR, $\mathrm{C} \times 1$ & 1.97 & - \\
\hline 6 & 75 & $\mathrm{M}$ & 3 & $\mathrm{~N}$ & CAD & 3 & 1 & 4 & $\mathrm{Y}$ & $\mathrm{C} \times 4$ & 2.02 & - \\
\hline 7 & 57 & $\mathrm{~F}$ & 1 & $\mathrm{~N}$ & $\begin{array}{l}\text { Rheumatic valve } \\
\text { disease }\end{array}$ & 1 & 3 & 3.8 & $\mathrm{~N}$ & M-VR & 3.03 & - \\
\hline 8 & 49 & M & 2 & $\mathrm{Y}$ & $\begin{array}{l}\text { Marfan syndrome } \\
\text { with dissection }\end{array}$ & 1 & 1 & 3.4 & $\mathrm{~N}$ & Redo $\times 5$ TAA & 6.50 & 481 \\
\hline 9 & 69 & M & 1 & $\mathrm{~N}$ & Calcific & 1 & 3 & 3.5 & $\mathrm{~N}$ & $\begin{array}{l}\text { A-VR, C } \times 1 \text {, } \\
\text { SMy }\end{array}$ & 2.63 & 359 \\
\hline 10 & 67 & $\mathrm{~F}$ & 2 & $\mathrm{~N}$ & PAD & 1 & 4 & 3.4 & $\mathrm{~N}$ & MT-VR, $\mathrm{C} \times 1$ & 4.10 & 525 \\
\hline 11 & 77 & M & 2 & $\mathrm{~N}$ & Calcific & 1 & 4 & 3.2 & $\mathrm{Y}$ & $\begin{array}{c}\text { AM-VR, } \mathrm{C} \times 2, \\
\text { AAA, Abl }\end{array}$ & 4.55 & 368 \\
\hline 12 & 80 & $\mathrm{~F}$ & 2 & $\mathrm{~N}$ & Calcific & 1 & 4 & 3.7 & $\mathrm{~N}$ & $\begin{array}{l}\text { A-VR, root } \\
\text { enlarge }\end{array}$ & 3.33 & 537 \\
\hline 13 & 39 & $\mathrm{~F}$ & 3 & $\mathrm{Y}$ & $\begin{array}{c}\text { Adult congenital } \\
\text { heart disease }\end{array}$ & 1 & 4 & 4.1 & $\mathrm{~N}$ & M-VR, Abl & 4.43 & 704 \\
\hline 14 & 57 & $\mathrm{~F}$ & 1 & $\mathrm{Y}$ & HOCM & 1 & 3 & 3.8 & $\mathrm{~N}$ & $\begin{array}{l}\text { M-VR, SMy, } \\
\quad \mathrm{C} \times 1\end{array}$ & 2.98 & 583 \\
\hline 15 & 62 & $\mathrm{~F}$ & 2 & $\mathrm{~N}$ & $\begin{array}{l}\text { Rheumatic valve } \\
\text { disease }\end{array}$ & 1 & 3 & 3.7 & $\mathrm{~N}$ & M-VR, Abl & 4.35 & 833 \\
\hline 16 & 80 & $\mathrm{~F}$ & 2 & $\mathrm{~N}$ & Mitral prolapse & 1 & 3 & 3 & $\mathrm{~N}$ & $\mathrm{M}-\mathrm{VR}$ & 3.15 & 431 \\
\hline 17 & 65 & $\mathrm{M}$ & 4 & $\mathrm{~N}$ & Calcific & 3 & 3 & 3.7 & $\mathrm{Y}$ & AM-VR & 4.43 & 789 \\
\hline 18 & 73 & $\mathrm{~F}$ & 1 & $\mathrm{~N}$ & Mitral prolapse & 1 & 4 & 3.7 & $\mathrm{Y}$ & M-VR, Abl & 3.10 & 457 \\
\hline 19 & 84 & M & 2 & $\mathrm{Y}$ & $\begin{array}{l}\text { Rheumatic valve } \\
\text { disease }\end{array}$ & 1 & 3 & 3.5 & $\mathrm{Y}$ & MT-VR & 3.30 & 741 \\
\hline 20 & 84 & $\mathrm{~F}$ & 2 & $\mathrm{~N}$ & Calcific & 1 & 4 & 3.6 & $\mathrm{Y}$ & A-VR, $C \times 3$ & 3.58 & 605 \\
\hline Mean & 73.0 & $55 \%$ & 3.0 & $25 \%$ & - & \multicolumn{2}{|c|}{$95 \%$ NYHA } & 3.5 & $50 \%$ & $95 \%$ complex & 3.83 & 543.0 \\
\hline SD & 15.6 & $\mathrm{~F}$ & 1.4 & $\mathrm{Y}$ & - & \multicolumn{2}{|c|}{ Class 3 or 4} & 0.1 & $\mathrm{Y}$ & Valve/AA & 0.35 & 87.7 \\
\hline
\end{tabular}

$A$, Aortic; $A A$, aortic aneurysm; $A A A$, ascending aortic replacement; $A b l$, atrial ablation; $A M$, aortic-mitral; $C$, coronary bypass; $C A D$, coronary artery disease; $H O C M$, hypertrophic obstructive cardiomyopathy; $I g$, immunoglobulin; $M$, mitral; $M T$, mitral tricuspid; NYHA, New York Heart Association; $P A D$, pure mitral annular dilatation; $S D$, standard deviation; SMy, septal myectomy; $T A A$, thoracoabdominal aneurysm; $V R$, valve repair or replacement.

IVIG administration was associated with significant improvement in thrombocytopenia $(P<.001)$, as evidenced by an increase in platelet count toward normal over the course of immune modulation (Figure 1). No limb ischemia was observed in any patient. Despite serious baseline profiles at day 0,19 of 20 patients $(95 \%)$ recovered uneventfully to hospital discharge. No significant complications of IVIG therapy occurred. Several patients experienced fever during drug infusion, and the administration rate was slowed. ${ }^{19}$

In 1 patient with $\mathrm{pT}$ who demonstrated more prominent TTP-like features (schistocytes, hemolysis, and a negative heparin PF4 antibody test result), plasmapheresis also was used over 3 days, with a prompt clinical response. This patient received extra doses of Ig to compensate for losses due to plasma exchange. Pulmonary cultures were positive in less than one third of patients, usually with antibioticresistant gram-negative organisms. ${ }^{12}$ The 1 patient who died had an initial good response, but then progressive sepsis developed and the patient died. Although recovery of serum creatinine was prolonged in several patients with acute renal failure, all surviving patients at some point recovered renal function to preoperative levels. ${ }^{12}$ One patient (\#15) did not receive the full 5-day course of IVIG. When his platelet count rebounded after 2 days of therapy, the drug was discontinued as a cost-savings effort. However, this approach then was attempted in the next 2 patients, and after they relapsed, IVIG was restarted and the full dose was completed successfully. By that time, an excellent meta-analysis had become available indicating that mortality improvement was better with full-dose and duration IVIG therapy, ${ }^{20}$ and limited doses were not attempted again.

\section{DISCUSSION}

In 1946, investigators at Harvard Medical School successfully fractionated human serum into protein and lipoprotein fractions using $8 \%$ ethyl alcohol. ${ }^{21}$ Then, with lowering $\mathrm{pH}$, gamma globulin could be extracted but caused severe reactions when administered intravenously. In 1962, enzymatic treatment of the purified Ig improved tolerance 


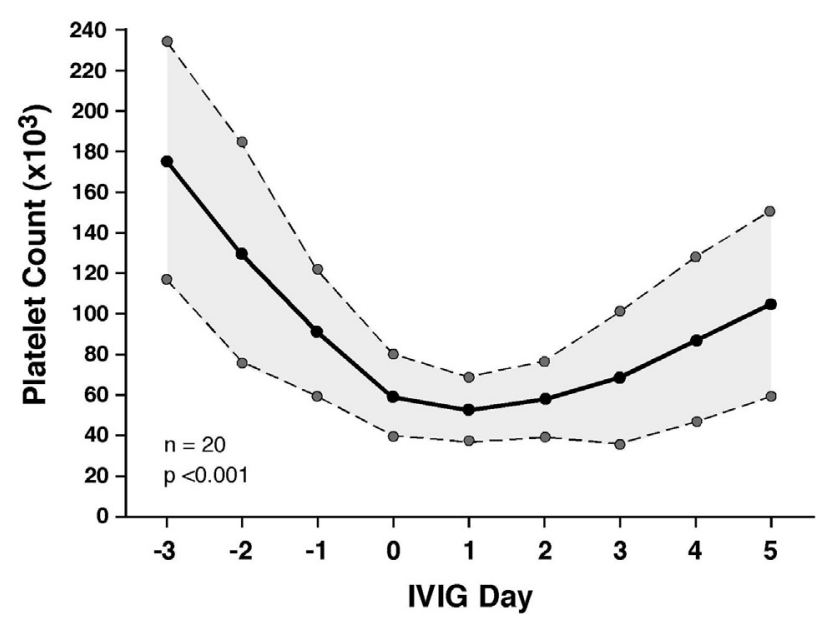

FIGURE 1. Mean \pm standard deviation of platelet counts for 3 days before $(-3)$ to 5 days after $(+5)$ beginning IVIG therapy in 20 postoperative patients experiencing pT. IVIG therapy was begun on day zero. Time course of the therapeutic response was assessed using a linear regression and spline function, with a statistical knot at day 0 , coincident with starting IVIG. IVIG was associated with a consistent reversal of developing $\mathrm{pT}$ and a highly significant treatment effect, using each patient as his or her own control $(P<.001)$. See text for details. IVIG, Intravenous immunoglobulin.

but weakened activity. In 1980 at laboratories in Bern, Switzerland, biochemical techniques were perfected to produce fully active Ig antibodies for intravenous administration, labeled IVIG at that time. ${ }^{21}$ The preparation contains pooled antibodies from 10 to $60 \mathrm{~K}$ plasma donations and manifests several million types of natural antibodies with a wide variety of functions. As a blood product, IVIG is sterilized by viral inactivation methods and nanofiltration, and with proper production techniques, IVIG has never caused a documented blood-borne infection after millions of doses. Soon after development, the immunology group in Bern used IVIG to treat $\mathrm{pT}$ in several children with immunemediated thrombocytopenia and documented successful results. Subsequent randomized trials confirmed the safety and efficacy of IVIG for treatment of immune-mediated thrombocytopenia, and IVIG has become an on-label firstline therapy for this disorder. IVIG use has increased from $300 \mathrm{~kg}$ per year in 1980 to 100 tons per year in 2010. More than 34,000 articles are listed in PubMed on IVIG and its immunomodulatory effects. ${ }^{21}$

So why has IVIG not been recognized as a valid treatment for postcardiac surgical pT? One reason is that we really do not understand the mechanisms of $\mathrm{pT}$ in the postoperative patient. Although heparin PF4 antibodies have been involved to some extent, it is unlikely that heparin antibodies are common etiologic factors. ${ }^{1}$ Because heparin antibodies develop in up to $40 \%$ of cardiac surgical patients, their presence is not specific enough in any given patient to fully implicate them. Moreover, most studies have not found a high incidence of PF4 antibody positivity, prompting consideration of other immune mechanisms. Some have proposed TTP-like antibodies, but evaluations of ADAMTS-13 moieties have been negative. ${ }^{3}$ Specific antibody surveys in surgical patients with pT could be revealing, and detailed immunologic research in the surgical setting is clearly needed. Infectious superantigens or gram-negative endotoxins are capable of inducing platelet aggregation and peripheral platelet deposition, similar to Escherichia coli Shiga toxin. ${ }^{7}$ Because all of our patients had signs of postoperative infection, we favor an infectious cause, most likely nosocomial pneumonia with predominantly hospital acquired gram-negative organisms. Whatever the pathophysiology, the uniform temporal response to immune modulation in our series would suggest immune-mediated causes. In fact, the immediate cessation in platelet count decline and the 1- to 3-day time lag to increasing platelets are suggestive of a time-dependent immune process. Although a more complete discussion has been published, ${ }^{22,23}$ it is clear that immune function represents a complex interrelated system and that IVIG is effective in suppressing inappropriate inflammatory responses and in restoring immunologic homeostasis, probably by multiple mechanisms.

Previous treatment modalities for pT left much to be desired. Although results have varied, anticoagulation with thrombin inhibitors have been associated with a residual incidence of limb loss, multiorgan failure, and death. Bleeding complications in early postoperative patients also have been problematic in some experiences. Plasmapheresis recently has been highly efficacious and probably removes offending autoantibodies in a rapid fashion. This intervention was effective in 1 of the patients in this series, and we have used it successfully twice subsequently. At this point, it is reasonable to propose inducing immune "normalization" with plasmapheresis (especially in patients with dialysis catheters in place or if the response to IVIG is slow) and then continuing immunomodulation with IVIG. Although some patients with rapidly decreasing platelet counts may not have immune-mediated pT (patient 15 was atypical with a normal IgG level and responded to 2 doses), we believe the protocol should be applied consistently to prevent the devastating complication of limb thrombosis (especially because IVIG complication rates are negligible). At some point, proper diagnostic tests may become available for immune-mediated $\mathrm{pT}$, but until then, routine application seems justified.

IVIG has been implicated in causing renal dysfunction when given in high doses. However, at the dose schedule used in this series, complications have not been observed, and all patients with developing renal failure eventually recovered baseline renal function. ${ }^{12}$ Never before have we had a method of directly treating postoperative renal failure, which in our experience almost always suggests developing 
infection. Other complications of IVIG were not observed, and the $5 \%$ of patients experiencing febrile responses were easily managed by slowing infusion. IVIG obviously is an expensive drug, and a full course costs $\$ 4$ to $\$ 8 \mathrm{~K}$, depending on the hospital's contracting mechanism. However, IVIG is likely cost-effective, considering that patients with thrombin inhibitor therapy had a mean hospitalization of 38 days in a recent series, ${ }^{1}$ whereas because of rapid clinical recovery, half of patients receiving IVIG were discharged after the fifth and last dose. ${ }^{12}$

In the final analysis, the best treatment for this disorder would be prevention. A good candidate for primary cause is nosocomial pneumonia, which is increasing nationally. ${ }^{24}$ Multiple interventions exist to reduce postoperative pulmonary infections, ${ }^{25}$ and better maintenance of operating room ventilators could be important. The aqueous environment of an unmaintained volume ventilator is ideal for gram-negative organisms, and in most major US centers, the devices are never cleaned. This is in contrast to the meticulous ventilator maintenance performed at German heart centers, in which postoperative pneumonia and $\mathrm{pT}$ are uncommon. ${ }^{26}$ Another recently defined nosocomial infection source is contaminated intravenous solutions. ${ }^{27}$ The revelation that cardioplegia solutions produced by the New England Compounding Center and used by many US hospitals were contaminated by black mold (Exserohilum rostratum) changes the meaning of the word "nosocomial." A major epidemiologic effort needs to be expended in US hospitals to reduce perioperative infection sources.

Most surgical patients in whom postoperative immune dysfunction develops are undergoing complex valve procedures (Table 1). ${ }^{12}$ They often are elderly and debilitated, frequently are emergency cases, and have longer cardiopulmonary bypass times. In a previous study of the first author's series, ${ }^{28}$ various manifestations of immune dysfunction developed in $18 \%$ of valve surgery cases and were treated with IVIG. Compared with earlier patients $(n=370)$ undergoing valve surgery, patients undergoing valve surgery with the backup of an IVIG protocol $(\mathrm{n}=171)$ had sicker baseline profiles, probably because of the later time period. Because immune dysfunction always has been an important cause of postoperative morbidity and mortality (perhaps the most important), effective management with IVIG decreased overall valve surgery mortality from $7.3 \%$ before the IVIG protocol to $1.2 \%$ after IVIG availability. Thus, a postoperative immune management protocol applied with highly selective criteria seems effective in reducing overall operative mortality ${ }^{29}$ and limiting morbidity associated with pT.

\section{Study Limitations}

IVIG therapy was not randomized, and most drug studies require randomized trials to ensure absence of treatment selection bias or confounding variables. Although this is a valid consideration, the concept of IVIG efficacy for immune-mediated $\mathrm{pT}$ has been established on multiple occasions, and the therapeutic response has been so consistent that the observation seems strong. In addition, the spline function analysis, using each patient as his or her own control, is a powerful assessment, suggesting temporal cause and effect rather than an association as in a randomized trial. ${ }^{30}$ Lack of patient randomization remains a limitation, although at the current level of experience, a randomized trial may be unethical.

\section{CONCLUSIONS}

IVIG administered to patients experiencing pT after cardiac surgery promptly and effectively reversed deteriorating platelet counts without major complications. Immune augmentation with IVIG offers the exciting possibility of effectively treating one of the significant problems encountered in cardiac surgical practice today and improving postoperative results. Studies of immunomodulatory mechanisms seem indicated.

\section{References}

1. Demma LJ, Paciullo CA, Levy JH. Recognition of heparin-induced thrombocytopenia and initiation of argatroban therapy after cardiothoracic surgery in the intensive care unit. J Thorac Cardiovasc Surg. 2012;143:1213-8.

2. Gurbuz AT, Elliott WG, Zia AA. Heparin-induced thrombocytopenia in the cardiovascular patient: diagnostic and treatment guidelines. Eur J Cardiothorac Surg. 2005;27:138-49.

3. Saltzman DJ, Chang JC, Jimenez JC, et al. Postoperative thrombotic thrombocytopenic purpura after open heart operations. Ann Thorac Surg. 2010;89:119-24.

4. McCormick JK, Yarwood JM, Schlievert PM. Toxic shock syndrome and bacterial superantigens: an update. Annu Rev Microbiol. 2001;55:77-104.

5. Norrby-Teglund A, Kaul R, Low DE, et al. Evidence for the presence of Streptococcal-superantigen-neutralizing antibodies in normal polyspecific immunoglobulin G. Infect Immun. 1996;64:5395-8.

6. Moake JL. Mechanisms of disease: thrombotic microangiopathies. N Engl J Med. 2002;347:589-600.

7. Tzipori S, Sheoran A, Akiyoshi D, et al. Antibody therapy in the management of shiga toxin-induced hemolytic uremic syndrome. Clin Microbiol Rev. 2004;17: 926-41.

8. Warkentin TE, Greinacher A. Heparin-induced thrombocytopenia and cardiac surgery. Ann Thorac Surg. 2003;76:2121-31.

9. Welsby IJ, Um J, Milano CA, et al. Plasmapheresis and heparin reexposure as a management strategy for cardiac surgical patients with heparin-induced thrombocytopenia. Anesth Analg. 2010;110:30-5.

10. Lakshmanan S, Cuker A. Contemporary management of primary immune thrombocytopenia in adults. J Thromb Haemost. 2012;10:1988-98.

11. CSL Behring LLC. Carimune NF, Nanofiltered Immune Globulin Intravenous (human). Prescribing Information. Kankakee, IL. Revised October 2008.

12. Rankin JS, Oguntolu O, Binford RS, et al. Management of immune dysfunction after adult cardiac surgery. J Thorac Cardiovasc Surg. 2011;142:575-80.

13. Greinacher A, Liebenhoff U, Kiefel V, et al. Heparin associated thrombocytopenia: the effects of various intravenous IgG preparations on antibody mediated platelet activation - a possible new indication for high dose i.v. IgG. Thromb Haemost. 1994;71:641-5.

14. Grau E, Linares M, Olaso MA, et al. Heparin-induced thrombocytopeniaresponse to intravenous immunoglobulin in vivo and in vitro. Am J Hematol. 1992;39:312-3.

15. Nurden AT, Laroche-Traineau J, Jallu V, et al. Heparin-induced thrombocytopenia: observation of the nature of the antibody activities and on the use of gammaglobulin concentrates in a patient with thrombotic complications. Thromb Haemost. 1991;65:796-8. 
16. Kress HG, Scheidewig C, Schmidt H, et al. Reduced incidence of postoperative infection after intravenous administration of an immunoglobulin A- and immunoglobulin M-enriched preparation in anergic patients undergoing cardiac surgery. Crit Care Med. 1999;27:1281-7.

17. Martin C, Ofotokun I, Rapp R, et al. Results of an antimicrobial control program at a University Hospital. Am J Health Syst Pharm. 2005;62:732-8.

18. Rapp RP. University of Kentucky Guide to Empiric Antimicrobial Therapy. Lexington, KY: University of Kentucky Press; 2002.

19. Bonilla FA. Intravenous immunoglobulin: adverse reactions and management. J Allergy Clin Immunol. 2008;122:1238-9.

20. Turgeon AF, Hutton B, Fergusson DA, et al. Meta-analysis: intravenous immunoglobulin in critically ill adult patients with sepsis. Ann Intern Med. 2007; 146:193-203.

21. Imbach P. Treatment of immune thrombocytopenia with intravenous immunoglobulin and insights for other diseases: a historical review. Swiss Med Wkly. 2012;142:1-10.

22. Rankin JS, Glower DD, Teichmann TL, et al. Immunotherapy for refractory pulmonary infection after adult cardiac surgery: immune dysregulation syndrome. J Heart Valve Dis. 2005;14:783-91.

23. Schwab I, Nimmerjahn F. Intravenous immunoglobulin therapy: how does IgG modulate the immune system? Nat Rev Immunol. 2013;13:176-89.

24. Lee R, Li S, Rankin JS, O'Brien SM, et al. Fifteen-year outcome trends for valve surgery in North America. Ann Thorac Surg. 2011;91:677-84.

25. Labelle A, Kollef MH. Healthcare-associated pneumonia: approach to management. Clin Chest Med. 2011;32:507-15.

26. Mazzitelli D, Noebauer C, Rankin JS, et al. Hemodynamic outcomes of geometric ring annuloplasty for aortic valve repair: a four-center pilot trial. J Thorac Cardiovasc Surg. 2013 Oct 8 [Epub ahead of print].

27. Additional contamination identified in medical products from New England Compounding Center. Posted in News, Outbreak Investigation, Centers For Disease Control And Prevention (CDC), Meningitis, Food And Drug Administration (FDA). December 21, 2012. Available at: http://www. infectioncontroltoday.com/news/2012/12/additional-contamination-identifiedin-medical-products-from-new-england-compounding-center.aspx. Accessed November 6, 2013.

28. Rankin JS. Effects of treating postoperative immune dysfunction on operative mortality after cardiac valve surgery. Abstract presented at Society Heart Valve Dis meeting, Barcelona, Spain, June 2011. Available at: http://www.jsrmd. com/ftp/67_IVIGV.avi. Accessed November 6, 2013.

29. Rankin JS, Burrichter CA, Walton-Shirley MK, et al. Trends in mitral valve surgery: A single practice experience. J Heart Valve Dis. 2009;18:359-66.

30. Harrell FE Jr. Regression Modeling Strategies: With Applications to Linear Models, Logistic Regression, and Survival Analysis. New York, NY: Springer Verlag, Inc; 2001

\section{Discussion}

Dr Nevin Katz (Baltimore, Md). This is an important report. This study addresses the important clinical issue of thrombocytopenia, which is not unusual in this current era of cardiac surgery in patients who have undergone complex cardiac surgery or who have ongoing infection or multiorgan system dysfunction postoperatively. The results of IVIG in your patients is impressive.

My first question regards the limitation of the study that you have recognized, and you mentioned that it is a nonrandomized study. There is no control group.

During the study period, did you encounter patients who developed postoperative thrombocytopenia of less than 60,000 but did not choose to receive the IVIG. If you did, could you comment on the courses of their platelet counts, and if present, the courses of their respiratory, renal, and infectious issues?

Dr Rankin. I am troubled by the limitations of randomized trials, and I have been involved with many of them over the years. They are good for some things, and studies that involve drugs usually require randomized trials for on-label approval. There have been many randomized trials using IVIG to modulate immune thrombocytopenia, and they all have been positive. It works.

In regard to nontreated patients, I did not have anyone who refused IVIG during this period. I was thinking, as you were talking, back to 1970, and my internship at Alabama (your former institution). At that time, I saw my first patient whose toes turned black after mitral valve surgery, associated with thrombocytopenia and multiorgan failure. So this disorder has been around for a while, and it has been one of the most frustrating problems that we encounter.

Then in the 1980s, Chuck Greenberg and Jim Lowe's first publication on heparin-associated thrombocytopenia thrombosis syndrome had 2 of my patients in it from Duke, and we would see this problem not infrequently in that era and since. So I have had a lot of experience with this syndrome over the years, and I have to say that since 2002 and institution of the IVIG protocol, it has been a relief to have zero patients with major complications from it. IVIG has been effective in every single patient experiencing a rapidly decreasing platelet count and immune dysfunction. Sometimes it takes several days to turn around, and I have 3 patients now in whom plasmapheresis was used in a complementary fashion in the first few days of therapy.

I am not sure that, at this point, a randomized trial would be ethical, given all the previous data with IVIG and immune thrombocytopenia. Indications are that the surgical variety is just another form of immune dysfunction like all the medical forms. The immune moiety, or inappropriate autoantibody, probably is going to be different. We need to explore that in basic immunologic research. I think it is clear that this problem is immune-mediated, and modulation with IVIG seems safe and extremely effective in the surgical setting.

Dr Katz. My second question has to do with the mechanism, and I think it is one of the important points of this report is detailing the various causes that could explain this. Because many people have thought that if the PF4 antibody test is negative, then it is not "HIT." So it is important to look at the mechanism.

Now, the fact that all but 1 patient in your series underwent valve or aortic surgery raises the question whether the decreased platelet counts were related to mechanical trauma to the platelets by passage of blood through the cardiotomy suction system of the pump oxygenator. I am wondering if you consider trauma related to heart-lung machines as a factor, and can you relate that to the immunologic mechanism?

Dr Rankin. It is unclear exactly what is going on there. If we look at our larger series of immune dysfunction, the factors that are common to these patients include worse baseline profiles, older sicker people, and longer pump runs. Pre-existing immune disorders, such as rheumatoid arthritis or lupus, also are common.

But it is mostly patients with complex valves, multiple valves in the elderly population, and so on, who get this syndrome. In a quick 1-hour pump run or 1.5-hour pump run for a coronary bypass, it is less common.

I believe it is more related to the duration of cardiopulmonary bypass and the degree of dysregulation of the immune system. Since we have been obtaining IgG levels postoperatively, the majority of these patients have low postoperative IgG levels. Sometimes, it will be 200 or 300 with a lower limit of 650 . It is hard to know cause and effect, because some infections are known to consume IgG, but low 
IgG levels certainly seem to be a marker. If the $\operatorname{IgG}$ level is 800 to 1000 after surgery, the patient rarely gets this problem. It is related, I think, to immune dysregulation contributed to in a major way by cardiopulmonary bypass. With nosocomial infection sources, immune depletion predisposes to the development of pulmonary infection. I believe most of these patients have gram-negative nosocomial pneumonias as their underlying problem.

I submitted an abstract 15 years ago saying that HIT was a major factor in postoperative mortality because $25 \%$ of the patients who died had thrombocytopenia syndrome, and I proposed that HIT then caused pulmonary dysfunction, pulmonary infiltrates, and multiorgan failure. Now, I am sure it is the other way around, that it is mostly infection-related. If I go to Germany where they maintain their operating room ventilators well, postoperative pneumonia is unusual. It might help US centers if they studied German infection control protocols.

Dr Katz. That is an interesting observation.

Dr Rankin. I do not have data on that; it is just my personal impression.

Dr Katz. Yes, but an interesting observation.

Dr Rankin. Yes.

Dr Katz. My final question. In this era of cardiac surgery, it certainly is not unusual to see platelet counts less than 60,000, and there are 3 groups of patients, at least in our practice, that I think I can identify in whom it is common. One group includes patients who have undergone reoperative surgery and have a coagulopathy postoperatively often related to perioperative antiplatelet therapy. The second group includes patients who are on ventricular assist devices and are undergoing extracorporeal membrane oxygenation. The third group includes patients who are being treated for sepsis postoperatively. On the basis of your study, would you recommend that IVIG be used in these groups of patients to decrease the need for platelet transfusions and improve outcomes related to multiorgan system dysfunction?

Dr Rankin. Prophylactically?

Dr Katz. Well, if you see the platelet count go down.

Dr Rankin. Yes.

Dr Katz. To maybe not 60,000, but you are seeing it go down, let's say, to less than 100,000, and you have someone on a ventricular assist device, do you think that it is appropriate to do that, or would you interpret the platelet count going down because of the system, the ventricular assist device or the extracorporeal membrane oxygenation system?

Dr Rankin. I do not have experience with the population with ventricular assist devices, and all the patients I reported today are probably in your third category with infection as the cause. IVIG works there, but I do not have experience with assist devices.

Certainly, there were patients in this series who experienced bleeding postoperatively and had transfusions, which probably is one risk factor, as we have discussed, but platelet counts were all restored to normal before the $\mathrm{pT}$ developed.

Dr Harold Burkhart (Rochester, Minn). Did you look into IgG levels before and after? Did you check that? I may have missed it.

Dr Rankin. Yes. Halfway through this series, around 2006, we obtained the ability to get IgG levels after surgery. As I said, the majority have low IgG levels immediately after surgery.

Dr Glenn Whitman (Baltimore, $M d$ ). I thought I saw that there were no positive cultures in this population, and yet I also thought I heard you are attributing thrombocytopenia in this population to Gram-negative septicemia. Could you explain the relationship further?

Second, would you suggest that every patient who has Gram-negative septicemia with thrombocytopenia be treated with IVIG whether or not they had cardiac surgery?

Dr Rankin. Regarding your second question, infection-related thrombocytopenia, from my interpretation of the literature, is an on-label indication for IVIG at present, for example, Shiga toxin cases. About your first question, I don't think I said there were no positive cultures. I said there were no patients with positive PF4 antibodies. But our particular hospital has been a little inconsistent with that test; we still have to send it to a remote laboratory, so my confidence in the PF4 results is not perfect. We were actually sending heparin-induced platelet aggregation studies to Duke for Greenberg's laboratory for a while, but again, the majority were not positive. In fact, in most publications, it has been unusual to have a positive PF4 in this population, usually with less than one third of patients exhibiting PF4 positivity.

In our published series of immune dysfunction in the Journal in 2011, approximately one third of these patients had positive pulmonary cultures, usually with Gram-negative rods, usually multidrug resistant. As you know from the literature, it is difficult to culture these kinds of organisms, with $30 \%$ to $40 \%$ positivity being fairly standard around the world. I believe most of these problems probably are related to postoperative pulmonary infection of one sort or another.

Dr Lyle Joyce (Rochester, Minn). So are all patients candidates or are there some patients who you would not treat?

Dr Rankin. Well, yes. Now, in a way, it is easier. I have stopped ordering PF4s; up to $40 \%$ of patients eventually turn positive after surgery anyway, so it is not a specific test. If the patient is 2 or 3 days postoperative, the IgG level is low, and the platelet count is plummeting, I just start the IVIG protocol. It is very important to complete the entire protocol $(1.5 \mathrm{~g} / \mathrm{kg}$ intravenously over 5 days) and not just a dose or 2 . It seems clear to me that most of the likely causes of pT are immune-mediated in one way or the other, and they all seem to respond to IVIG in my series.

Dr Joyce. You will not wait for a negative hit, then?

Dr Rankin. Oh, no.

Dr Joyce. You just go ahead and get started?

Dr Rankin. Well, if it is a TTP-like antibody, as several articles propose, it works there. If it is endotoxin, it works there. If it is a PF4 antibody, IVIG is a standard therapy. The immune modulatory effects of IVIG are effective in all those settings, and it has been $100 \%$ effective in our population.

Dr Joseph Rubelowsky (Hattiesburg, Miss). This is a fascinating topic because I am dealing with this in exactly the same population you are talking about with multivalves and patients who develop renal failure. I round in the morning and say oh my, the platelet count is low.

Now that I have this in my armamentarium, I never considered this. But one thing I would be interested in is with my patient who recently had a low platelet count and vancomycin-resistant Enterococcus, and it was terrible. But I am always worried, and like you said, this happens in valve cases. Then I have transesophageal echocardiograms to show there were no vegetations or paravalvular leaks. 
When you looked at those patients, those 20 patients who went out, are they coming back with paravalvular abscesses or infective endocarditis, or do you keep them on long-term antibiotics just to make sure that if they were septic, that we killed that bug and they are not going to have a problem with that valve?

Dr Rankin. As we have written in our immune dysfunction articles in the Journal and The Journal of Heart Valve Disease, proper antibiotic coverage together with augmenting the immune system are both important.
So, it pays to obtain good bronchoalveolar cultures and use good antibiotic protocols. I am sure that secondary endocarditis can occur. I've had that happen in the remote past. But in this series, once the patients got over the pulmonary infection and their white blood counts returned to normal, the pT did not recur. I had 1 patient who required a second valve operation 1 week after receiving IVIG for serious thrombocytopenia, and with the IVIG on board, the thrombocytopenia did not recur. It looks to be an effective therapy in the postsurgical setting. 